Documentation et bibliothèques

\title{
La rotation automatisée des collections dans les bibliothèques centrales de prêt
}

\section{The automated rotation of collections in central lending} libraries

\section{La rotación automatizada de colecciones en las bibliotecas centrales de préstamo}

\section{Richard Boivin et Norman A. Fink}

Volume 34, numéro 4, octobre-décembre 1988

URI : https://id.erudit.org/iderudit/1051044ar

DOI : https://doi.org/10.7202/1051044ar

Aller au sommaire du numéro

Éditeur(s)

Association pour l'avancement des sciences et des techniques de la documentation (ASTED)

ISSN

0315-2340 (imprimé)

2291-8949 (numérique)

Découvrir la revue

Citer cet article

Boivin, R. \& Fink, N. A. (1988). La rotation automatisée des collections dans les bibliothèques centrales de prêt. Documentation et bibliothèques, 34(4), 143-156. https://doi.org/10.7202/1051044ar
Résumé de l'article

Les bibliothèques centrales de prêt ont établi, au cours des dernières années, un programme informatisé de rotation des livres, des disques, des cassettes audio et vidéo entre elles et les bibliothèques affiliées. Les auteurs présentent ici les objectifs du programme, résument les étapes de son implantation, définissent et explicitent les fonctions des divers modules et jettent un regard sur l'avenir.
Tous droits réservés (c) Association pour l'avancement des sciences et des techniques de la documentation (ASTED), 1988
Ce document est protégé par la loi sur le droit d'auteur. L'utilisation des services d’Érudit (y compris la reproduction) est assujettie à sa politique d'utilisation que vous pouvez consulter en ligne. 


\title{
La rotation automatisée des collections dans les bibliothèques centrales de prêt
}

\author{
Richard Boivin * \\ $\mathrm{BCP}$ de la Montérégie \\ Laprairie, Oc \\ Norman A. Fink * \\ $B C P$ de l'Abitibi-Témiscamingue \\ Noranda, Qc
}

Les bibliothèques centrales de prêt ont établi, au cours des dernières années, un programme informatisé de rotation des livres, des disques, des cassettes audio et vidéo entre elles et les bibliothèques affiliées. Les auteurs présentent ici les objectifs du programme, résument les étapes de son implantation, définissent et explicitent les fonctions des divers modules et jettent un regard sur l'avenir.

\section{The automated rotation of collections in central lending libraries}

The central lending libraries have developed an automated programme for rotating the books, records and audio and video cassettes among the central libraries and their affiliates. The authors present the objectives of the programme, describe the steps taken to set it up, define and explain the functions of its modules and provide a perspective for the future.

\section{La rotación automatizada de colecciones en las} bibliotecas centrales de préstamo

En los ùltimos años las bibliotecas centrales de préstamo han establecido entre ellas y las bibliotecas afiliadas un programa automatizado en cuanto a la rotación de libros, discos, audiocasetas y videocasetas. Los autores presentan en este artículo los objetivos del programa, exponen las etapas de su implantación, definen $y$ explican las funciones de los distintos módulos y echan una mirada al porvenir.
Les bibliothèques centrales de prêt ( $B C P)$ sont des sociétés privées sans but lucratif créées à l'instigation du ministère des Affaires culturelles du Québec (MAC). Elles ont pour but la mise sur pied et le soutien des bibliothèques des municipalités de moins de 5000 habitants d'une région donnée. Les onze BCP du Québec desservent 828 municipalités, soutiennent 5000 bénévoles qui oeuvrent au sein des divers comités de bibliothèques. Le réseau des BCP gèrent plus de deux millions de livres, disques, films, reproductions d'oeuvre d'art et vidéo-cassettes. Elles font annuellement cinq millions de prêts.

\section{L'addition et le partage des ressources}

Les BCP cherchent à développer leurs collections en acquérant le plus grand nombre de titres possible mais, compte tenu de leurs budgets retreints et des intérêts variés des lecteurs, elles doivent en limiter le nombre d'exemplaires. II est donc essentiel que toutes les bibliothèques, qui s'associent au réseau d'une BCP, additionnent et partagent leurs ressources dans le meilleur intérêt de leurs usagers.

\footnotetext{
* Richard Boivin est directeur général de la Bibliothèque centrale de prêt de la Montérégie et Norman A. Fink est directeur général de la Bibliothèque centrale de prêt de l'Abitibi-Témiscamingue.
} 
L'apport et le partage des ressources s'actualise principalement par l'échange périodique des collections déposées par la BCP dans les bibliothèques affiliées; il s'agit donc d'une gestion des collections et de la production d'outils bibliographiques tels que catalogues imprimés ou microfilmés. Le processus s'appelle la rotation des collections.

Le partage des ressources se concrétise aussi par le sous-système des demandes spéciales, lequel permet aux usagers l'accès à l'ensemble de la collection du réseau de la BCP. Ce sous-système est en quelque sorte un prêt entre bibliothèques affiliées.

Enfin pour compléter le processus de partage et I'apport des ressources, les bibliothèques affiliées développent localement des collections complémentaires à celles déposées par la BCP. Ces collections deviennent accessibles, par le service des demandes spéciales, à l'ensemble du réseau.

\section{Les expériences pilotes à la base du choix d'un système}

Dès le début de sa fondation, au milieu des années soixante-dix, la BCP de l'AbitibiTémiscamingue (BCPAT) constata la complexité et la répétition des opérations qu'exige le processus de rotation des collections. Elle fit donc, auprès du ministère des Affaires culturelles (MAC) du Québec, une demande de subvention afin de mener à bien un projet pilote d'automatisation des rotations.

Une étude réalisée à la demande du MAC par le Bureau central de l'informatique du ministère des Communications en 1979 a démontré la rentabilité de l'informatisation des opérations de rotations, des demandes spéciales et la publication de catalogues de collections de biens culturels dans les bibliothèques centralesjde prêt du Québec.

Avant de procéder à l'implantation d'un système d'information aux fins de gestion (SIG) dans l'ensemble des BCP, le ministère des Affaires culturelles et les BCP convenaient de procéder à la réalisation d'un projet pilote à la $\mathrm{BCP}$ de l'Abitibi-Témiscamingue, laquelle avait jusqu'alors mené le dossier. Les BCP s'entendaient pour partager les coûts des logiciels tandis que le MAC s'engageait à financer l'équipement requis. La BCPAT, pour sa part, s'occuperait de la réalisation du projet pilote.

À la demande de la BCPAT et du Service des programmes régionaux du MAC, le Bureau central de l'informatique $(\mathrm{BCl})$ du ministère des Communications évalua l'expérience pilote et déposa son rapport en novembre 1984. Les analystes du $\mathrm{BCl}$ concluaient que le système développé par la BCPAT démontrait les bénéfices de l'informatisation; que les autres BCP devraient, dès que possible, bénéficier des avantages de l'informatisation et enfin, que trois possibilités s'offraient au MAC et aux BCP: le statu quo, l'adoption du système développé à la BCPAT, ou la réalisation d'un nouveau système programmé en fonction des connaissances acquises au cours de l'expérience pilote.

Fort de l'évaluation du $\mathrm{BCl}$, le Regroupement des bibliothèques centrales de prêt du Québec adoptait une résolution demandant la réalisation, en 1985, d'un deuxième projet pilote d'informatisation. La bibliothèque centrale de prêt de la Montérégie fut chargée de la mise au point d'une version améliorée du système BCPAT. En plus de la correction des lacunes décelées par la $\mathrm{BCl}$, ce deuxième projet pilote devait mener à une réduction des coûts d'implantation, à une normalisation des données catalographiques et à l'utilisation maximum de l'ordinateur.

La BCP de la Montérégie a retenu en 1985 le système SIGIRD (système intégré de gestion informatisé des ressources documentaires) de I'Université du Québec à Montréal. L'UQAM venait d'en confier la commercialisation à la société Multitek du Groupe Sobeco.

Conçu et implanté dans un environnement réseau, on pouvait envisager son utilisation dans chaque bibliothèque affiliée à une bibliothèque centrale de prêt sans développement informatique majeur. En partant d'une base solide, on intégrait un module spécifique aux bibliothèques centrales de prêt: la rotation des collections. Le module des rotations était conforme au cahier de charges ayant servi à l'expérience pilote de la BCPAT avec les améliorations souhaitées dans le rapport du $\mathrm{BCl}$.

En jumelant le système des rotations de la BCPAT à SIGIRD, lequel allait devenir plus tard multiLIS, la BCP Montérégie se retrouvait avec un système d'avant-garde complet, intégré, souple, évolutif et fiable. Les BCP pourraient profiter des innombrables améliorations apportées aux différents modules du logiciel sans avoir à en assumer la totalité des coûts. Elles pouvaient donc consacrer la grande part de leurs efforts au développement du module des rotations.

Bénéficiant de l'expérience concluante de deux BCP, le Regroupement des BCP du Québec proposait en avril 1987 un programme global d'automatisation, à partir d'un logiciel commun à l'ensemble des $\mathrm{BCP}$, soit le système multiLISrotation. En vertu du décret 455-88, le gouvernement du Québec, sur la recommandation de la ministre des Affaires culturelles et du ministre 
délégué au Développement régional, adoptait, en mars 1988, un plan triennal d'automatisation des $\mathrm{BCP}$, en assumant le financement à $80 \%$ grâce à une subvention de 1344000 \$

C'est le Regroupement des BCP qui assume, en vertu d'une entente avec le MAC, la gestion du programme; ainsi, il a acquis pour l'ensemble des $\mathrm{BCP}$ une licence globale d'exploitation du système multiLIS. La BCP de la Mauricie (162 bibliothèques affiliées), celle des Laurentides (64) et celle des Portages ont débuté leur processus d'automatisation et sept autres l'implanteront d'ici mars 1991. Dès lors, ce sont plus de 900 bibliothèques publiques qui bénéficieront des banques de données ainsi que des outils d'exploitation des collections produits grâce à l'ordinateur.

\section{Qu'est-ce que la rotation des collections?}

La BCP dépose dans chacune des municipalités affiliées de son réseau un lor de biens culturels (livres, disques, oeuvres d'art, jouets) en quantité proportionnelle à leur population. C'est la bibliothèque affiliée qui effectue le prêt à la population.

Afin de maintenir l'intérêt des citoyens de chaque ville ou village qu'elle dessert, la BCP procède généralement à tous les quatre mois au renouvellement d'une partie de la collection déposée. Cette activité d'échanges des biens culturels entre la BCP et ses bibliothèques affiliées s'appelle la rotation des collections.

La collection mise en dépôt compte des caractéristiques propres à chaque milieu. II est nécessire d'assurer un équilibre basé sur l'âge de la population (proportion jeunes-adultes), le genre de documents (romans, documentaires, bandes dessinées, etc.) et la langue des usagers.

La collection déposée est composée de types de biens culturels tels que les volumes français (VOL), volumes anglais (BKS), disques et cassettes (DIS), etc. Chaque type de biens est divisé en catégories. Ainsi les volumes français le seront en documentaires pour adultes, en romans, en livres pour jeunes. Chaque catégorie peut être à son tour subdivisée en sous-catégories correspondant aux classes de Dewey pour les documentaires et en bandes dessinées, romans (jeunes), albums, documentaires (jeunes) pour les livres destinés aux jeunes.

Un pourcentage de la collection mise en dépôt est établi pour chaque catégorie ou souscatégorie. Lors de la rotation, cet équilibre doit être maintenu. De là, toute la complexité du processus lorsqu'une BCP doit assurer la rotation de 160 bibliothèques affiliées à raison de trois échanges par année. La précision du système ne pouvait être garantie que par son automatisation.

\section{MultiLIS/rotation des collections}

MultiLIS est un système intégré des fonctions documentaires. II permet le traitement réseau entre les bibliothèques affiliées et leur BCP ainsi qu'entre les BCP du Québec. Nous ne nous attarderons ici qu'au module de rotations.

Les fonctions de base du module de rotation sont essentiellement l'addition et le partage des ressources documentaires des bibliothèques du réseau d'une BCP. L'addition de ressources, c'est l'ajout d'une collection de volumes dans une bibliothèque affiliée. Le partage des ressources repose sur l'échange périodique d'une partie des volumes déposés initialement et sur les prêts par demandes spéciales.

II faut tenir compte également du fait que certaines bibliothèques affiliées aux $B C P$ développent leur propre collection à côté de celle de la BCP. Ces collections locales demeurent complémentaires à la collection de base fournie par la BCP et peuvent servir aux usagers de l'ensemble du territoire.

Le module de rotation des collections s'applique aux livres, disques, cassettes et oeuvres d'art. Mais afin de faciliter la compréhension du module nous ne tiendrons compte que des livres.

\section{Fonctions du module}

Le module des rotations fait partie intégrante du système multiLIS. On le retrouve donc au menu général (Voir figure 1). Le module des rotations des collections de multiLIS repose sur onze fonctions que nous décrirons sommairement.

\section{Gestion des bibliothèques affiliées}

La gestion des bibliothèques affiliées est un élément très important du module rotation. Le système considère comme bibliothèque affiliée un dépôt de biens culturels. Le magasin de la BCP ou une collection spéciale sont aussi considérés comme des dépôts à l'intérieur du système. Une bibliothèque affiliée peut offrir plusieurs types de biens culturels tels que volumes français, volumes anglais, disques, cassettes, oeuvres d'art, etc.

Plusieurs éléments d'information sont consignés au sous-module gestion des bibliothèques affiliées. Ces informations sont entrées ou 
modifiées à l'aide du "format " d'écran illustré à la figure 3. En voici les détails :

NUMÉRO DE BIBLIOTHĖQUE AFFILIÉE. Le système peut compter jusqu'à 225 bibliothèques affiliées. Le numéro assigné à la bibliothèque affiliée est unique et l'assignation se fait au fur et à mesure qu'une bibliothèque s'ajoute au réseau.

La bibliothèque affiliée zéro (0) est réservée au magasin de la BCP. Le système inscrit automatiquement toute nouvelle acquisition de la $B C P$ à la bibiliothèque affiliée zéro $(0)$ avec la mention disponible.

ADRESSE D'EXPÉDITION ET DE CONTACT. L'adresse d'expédition est l'adresse où les biens culturels doivent être livrés. Cette adresse correspond généralement à l'adresse de la bibliothèque. C'est à cette adresse qu'on fera parvenir les avis de rotation ou le courrier ayant trait à un échange de biens culturels.

INFORMATIONS COMPLÉMENTAIRES. Afin de pouvoir gérer efficacement l'ensemble du processus de rotation, il faut indiquer certaines informations qui serviront soit au système soit à l'opérateur.

\section{Responsable}

C'est la personne désignée comme telle par la municipalité. Elle supervise les activiés de la bibliothèque au niveau local et elle agit comme interlocuteur auprès de la BCP.

\section{Responsable des demandes spéciales (DS)}

Les demandes spéciales sont un genre de prêt entre bibliothèques (entre la centrale et ses bibliothèques affiliées). II n'y a pas de prêts directs entre les bibliothèques affiliées. Pour certaines bibliothèques, les demandes spéciales sont un service d'appoint important à la collection déposée et souvent on y affectera une personne autre que la responsable de la bibliothèque. II faudra donc bien identifier la personne désignée.

\section{Type de biens}

Une bibliothèque affiliée peut avoir une collection de volumes français, de disques et de cassettes, de jouets éducatifs, de cassettes vidéos et de volumes anglais.

\section{Collection locale}

La collection locale est l'ensemble des volumes et autres documents qui appartiennent à la bibliothèque affiliée. Les bibliothèques affiliées signalent à la BCP leurs achats et ils sont intégrés à sa banque de données. De la sorte, l'ordinateur tiendra compte de la collection locale et on évitera ainsi d'expédier des doubles.

\section{Remarques}

Ce champ permet de consigner des informations supplémentaires: bibliothèque affiliée fermée, responsable remplaçante, etc.

STATUT DE ROTATION. Le statut de rotation est mis automatiquement EN ATTENTE lorsqu'on crée une nouvelle bibliothèque. Par la suite, il peut afficher ACTIF ou INACTIF selon le cas. Une bibliothèque affiliée doit être active si on veut que l'ordinateur y inscrive des biens culturels en vue d'un échange. On ne peut ajouter des biens culturels à une bibliothèque considérée comme inactive que par le sous-module des demandes spéciales. Qu'elle soit active ou non, on peut retirer un bien culturel en tout temps d'une bibliothèque.

\section{Numéro de rotation}

Le numéro de rotation est mis automatiquement à zéro (0) lors de la création d'une nouvelle bibliothèque. II avancera de 1 chaque fois qu'on procèdera à une rotation de biens culturels. Le système garde en mémoire I'historique de neuf rotations.

\section{Nombre de rotation / année}

Le système peut supporter jusqu'à 24 rotations par année. Le nombre annuel de rotations est déterminé par chaque $\mathrm{BCP}$; cependant, on procède généralement à trois rotations par année. Le nombre de rotations qu'on indique à ce champ vaut pour l'ensemble des types de biens culturels. Ainsi il y aura rotations pour les volumes, les disques ou les livres anglais.

\section{Priorité de rotation}

L'opérateur du système assigne à une bibliothèque affiliée la priorité de rotation qu'il désire. Chaque poste de travail peut avoir ses propres priorités. II faut noter que le système inscrit les biens culturels dans l'ordre des priorités établies au niveau du poste de travail.

MODIFICATIONS. Naturellement dans un réseau où I'on compte les intervenants par centaines, il est important de pouvoir modifier rapidement l'information servant à la gestion du module. Cette fonction permet de mettre à jour le dossier de chaque bibliothèque affiliée. 


\section{Gestion des types de biens culture/s}

Pour indiquer au système le nombre de biens culturels que la BCP doit déposer dans une succursale il faut utiliser le sous-module Gestion des quantités par type de biens culturels.

TYPE DE BIENS CULTURELS. Lors du traitement d'un bien culturel, le système lui assigne automatiquement, à partir de l'indice de classification, un code de type de biens culturels. La zone 31 du bordereau de catalogage de multiLIS a été ajoutée à cet effet. Le type de biens culturels fait référence à une collection spécifique. Les principaux types de biens sont les volumes français (VOL), les volumes anglais (BKS) et les disques et cassettes (DIS).

L'entrée d'un nouveau type de biens culturels se fait par la fonction 6 du module de catalogage (gestion des dictionnaires). Le système peut inclure jusqu'à 99 types de biens culturels différents.

ÉQUILIBRE CONSTATÉ. L'équilibre constaté est la somme des équilibres des catégories appartenant à ce type de biens culturels. L'équilibre constaté peut donc être supérieur ou inférieur à 100.

Si l'équilibre est supérieur à 100 , c'est que la $B C P$ a expédié un plus grand nombre de documents dans une catégorie que voulu au départ. Si l'équilibre n'est pas corrigé, on échangera également un plus grand nombre d'unités pour ce type de biens culturels. Si l'équilibre est inférieur à 100, c'est l'inverse qui se produira.

II appartient à l'opérateur du système d'indiquer à l'ordinateur qu'il veut ramener l'équilibre à 100 . Le système fera les corrections nécessaires aux catégories identifiées.

QUANTITÉ GLOBALE. C'est la quantité totale d'unités d'un type de biens culturels déposées dans une bibliothèque affiliée. La quantité globale tient compte à la fois des biens culturels actuellement dans la bibliothèque, ajoutés ou soustraits par les demandes spéciales et en processus de rotation s'il y a lieu. Généralement la collection déposée correspond à un minimum d'un livre par tête. Les BCP déposent habituellement un minimum de 1000 volumes dans une bibliothèque même si la population du village est inférieure à 1000 habitants.

QUANTITÉ À AJOUTER. La quantité à ajouter est la quantité totale de biens culturels que l'on désire expédier à une succursale en dehors du processus normal de rotation. Ainsi, pour une nouvelle succursale on inscrira ici la quantité de biens culturels qui doit y être déposée pour l'ouverture. Cette quantité correspond habituellement à la population de la municipalité (village + paroisse).

Si on veut accroître la collection déposée originellement tout en conservant l'équilibre initial, c'est par cette fonction qu'on y parviendra. Toutefois, si on veut accroître ou réduire (s'il s'agit d'inscrire une quantité négative) la quantité de volumes déposés pour une seule ou quelques catégories, il faudra le spécifier. II est évident qu'en ajoutant des unités à une catégorie spécifique, I'on modifiera l'équilibre de départ pour cette catégorie.

TAUX DE ROTATION ANNUEL. Le taux de rotation annuel est déterminé par la BCP. Ce taux est pondéré par l'importance de la collection déposée selon chaque type de biens culturels. Ainsi pour une collection de 1000 livres, le taux pourrait être de $105 \%$ alors que pour une collection de 5000 livres il ne serait que de $48 \%$.

Lorsque le système calcule le nombre de livres à échanger, il divise le taux de rotation par le nombre de rotations annuelles. Un taux annuel de $105 \%$ donnera un roulement quadrimestriel de $35 \%$.

\section{Gestion des catégories par type de biens culturels}

Pour indiquer au système les catégories de biens culturels à l'intérieur d'un type de biens culturels, il faut utiliser le sous-module Gestion des catégories par type de biens culturels (voir Figure 5).

CATÉGORIES. Un type de biens culturels peut être subdivisé en plusieurs catégories. On retrouve, pour les livres de langues française et anglaise, 18 catégories; le documentaire est subdivisé selon les grandes classes de la classification Dewey $(100,200 \ldots)$; les livres de jeunes comprennent les catégories bandes dessinées, albums, documentaires et romans: enfin les romans pour adultes peuvent aussi être répartis en diverses catégories telles que romans policiers, sentimentaux ou d'aventure.

Bien entendu, le système permet une subdivision beaucoup plus élaborée. Comme il faut faire des échanges équilibrés comportant un certain pourcentage de nouveautés, on comprendra que la gestion du système peut devenir vite impossible tant pour le personnel affecté aux rotations que pour les bibliothèques affiliées. II y a lieu de limiter le nombre de rotations annuelles. II semble d'ailleurs que la presque totalité des BCP ait adopté trois rotations par année. 
ÉQUILIBRE DES CATÉGORIES. L'équilibre détermine la composition, en pourcentage, par catégorie de la collection de la bibliothèque affiliée; ce nombre doit être compris entre 0 et 100. II est calculé par le système et reflète la réalité. Par ailleurs, on peut contrôler ce paramètre en utilisant l'équilibre visé. Lorsqu'on indique zéro (0), on signifie au système qu'aucun bien de cette catégorie ne doit figurer dans la collection de la bibliothèque affiliée. Cent (100) signifie que la collection d'une bibliothèque doit être entièrement composée de documents de cette catégorie.

Le pourcentage d'une catégorie dans un type de biens culturels est déterminé selon l'importance qu'a cette catégorie dans la collection générale ou déposée, selon l'envergure de lajcollection, selon l'historique du prêt de cette catégorie et selon l'intérêt du public découlant des profils démographiques de la population.

Ainsi, une population de 1000 habitants et moins se verra octroyer un pourcentage plus élevé de romans pour adultes soit $30 \%$ comparativement à $20 \%$ pour une population de 5000 habitants. En fait, il faut tenir compte de la théorie du rendement marginal. II peut y avoir autant d'équilibres qu'il y a de bibliothèques affiliées et donc des collections adaptées aux profils de chaque bibliothèque. Voici un exemple d'équilibre désiré en fonction d'une bibliothèque donnée.

Types de biens : volumes français

- Catégorie des documentaires adultes : (30\%)

Sous-catégories

$0-99=1 \%, 100=4 \%, 200=.5 \%, 300=$

$3 \%, 400=.5 \%, 500=2 \%, 600=5 \%, 700$

$=3 \%, 800=2 \%, 900=2 \%, 920=5 \%, 930$

$=2 \%$.

\section{- Catégorie Romans adultes : (30\%)}

- Catégorie Livres jeunes : (40\%)

- Sous-catégorie Albums $=12 \%$, Romans $=$ $8 \%$, Bandes dessinées $=11 \%$, Documentaires $=9 \%$.

Même si la BCP préconise un certain équilibre pour une bibliothèque affiliée, il peut arriver que cet équilibre soit modifié par le jeu des demandes spéciales et des retours à la centrale. L'ordinateur indique donc à la fois l'équilibre constaté pour cette catégorie et l'équilibre désiré. Le préposé pourra rétablir la situation d'un échange à l'autre.

PÉRIODE DE VALIDITÉ DES NOUVEAUTÉS. LeS $B C P$ achètent les nouveaux titres en plusieurs exemplaires, lesquels demeurent toutefois limités par rapport au nombre de bibliothèques affiliées. Un livre paru dans l'année qui a été déposé dans une bibliothèque affiliée et qui revient lors d'un échange à la centrale après quatre mois est encore une nouvelle acquisition pour la prochaine bibliothèque où ce livre sera inscrit. Un délai est donc prévu pour que le système considère un livre comme nouveauté lors de l'inscription.

Cette période varie selon les catégories de livres. Ainsi, un roman sera considéré nouveauté pendant 18 mois tandis qu'un livre de la catégorie 200 (religion) le sera 36 mois. Si on décidait de modifier ces périodes de temps, les modifications n'affecteraient que les documents inscrits dans la banque après la date de modification.

PÉRIODE DE VALIDITÉ DU DÉPÔT. Afin d'éviter qu'un livre soit retourné prématurément à la bibliothèque affiliée, on a prévu une période pendant laquelle ce titre ne sera pas retourné à cette bibliothèque de façon automatique. Cette période varie selon la catégorie du document. À titre d'exemple, elle sera peut-être de 18 mois pour une bande dessinée et de 12 mois pour un roman.

TAUX DE ROTATION DE LA CATÉGORIE. Le tauX de rotation de la catégorie indique le taux annuel de renouvellement de cette catégorie au sein de la collection d'une bibliothèque affiliée. Zéro (0) signifie qu'il n'y a aucun renouvellement pour cette catégorie, donc aucun bien expédié ou à recevoir lors de l'échange. Cent (100) signifie que cette catégorie se renouvellera en entier après le nombre d'échanges indiqué pour une année. Le taux de rotation annuel peut être supérieur à $100 \%$ pour une catégorie.

Le taux indiqué est le taux annuel; I'ordinateur divisera ce taux par le nombre d'échanges prévus dans l'année. Ainsi, un taux de rotation annuel de $96 \%$ implique pour cette catégorie un taux de rotation de $32 \%$ par échange soit 96/3.

Le taux de rotation annuel peut varier d'une catégorie à l'autre. Comment s'assurer que la somme du taux de catégorie correspondra au taux global de rotation? Des critères tels que le prêt dans cette catégorie, le caractère éphémère de l'information, l'importance de la collection déposée doivent être évalués par la responsable de l'organisation des collections afin de déterminer un taux de rotation raisonnable. La somme des quantités échangées par catégorie ne doit pas dépasser le taux de rotation annuel pour l'ensemble des catégories d'un type de biens culturels déposés.

POURCENTAGE DES NOUVEAUTÉS. Le pourcentage des nouveautés indique pour la 
catégorie la proportion de nouveautés que l'on devrait expédier à cette succursale. Le pourcentage s'établit entre zéro (0) et cent (100).

Le pourcentage de nouveautés par catégorie est déterminé par le stock dont la BCP dispose pour cette catégorie et par les retours des bibliothèques affiliées. II est très difficile d'évaluer le nombre de biens culturels retournés dont la période de nouveauté n'est pas terminée lors d'un échange par une bibliothèque affiliée. II faut donc évaluer régulièrement les disponibilités de la BCP. Des outils tels que le tableau sur l'état des rotations, l'inventaire des collections déposées ainsi que l'expérience du personnel affecté aux rotations seront très utiles pour établir le pourcentage de nouveautés par catégorie.

Toutefois, il est possible d'indiquer que le pourcentage de nouveautés pour certaines catégories soit obligatoire ou facultatif. Dans le cas "obligatoire", le système refusera d'inscrire le livre à la bibliothèque affiliée s'il n'est pas unejnouveauté ou si le nombre voulu est atteint. Quant au "facultatif", si le nombre de livres anciens est atteint pour cette catégorie, il inscrira le volume dans la catégorie nouveauté qu'il en soit une ou non. Par un écran de visualisation, l'opérateur peut savoir si l'assignation facultative des nouveautés est accesptable quant à la répartition anciens-nouveaux.

COLLECTION LOCALE. À ce niveau, on indique au système si lors de l'inscription, l'ordinateur doit tenir compte de la collection locale de la bibliothèque affiliée. Rappelons que la collection locale de la bibliothèque doit être traitée au préalable sur l'ordinateur de la BCP pour que l'on en tienne compte lors de l'inscription. L'ordinateur refusera d'expédier le livre si la bibliothèque affiliée le possède déjà.

\section{Initialisation de la rotation}

Comme nous visons une optimisation de I'utilisation de l'ordinateur et que ceci est impossible avec 50, 100 ou 160 bibliothèques, on rendra une bibliothèque active uniquement si elle est prévue prochainement au calendrier des échanges hebdomadaires ou si elle est nouvellement affiliée au réseau. L'ordinateur n'inscrit des livres que dans les bibliothèques considérées comme actives.

La date et l'heure de l'échange indiquent le moment de la livraison à la bibliothèque affiliée. Un avis d'échange sera produit afin d'informer la responsable de la bibliothèque de la quantité de biens culturels à retourner et de celle que lui expédiera la centrale.

\section{Inscription des biens culturels}

Cette fonction contrôle le processus par lequel on désinscrit et assigne des biens culturels à des bibliothèques affiliées préalablement à l'expédition de ces biens.

MODE D'INSCRIPTION. Le mode d'inscription peut être automatique ou manuel. En mode automatique, c'est l'ordinateur qui choisit la destination tandis qu'en mode manuel, c'est le préposé. Peu importe le mode, l'ordinateur affiche à l'écran certaines informations abrégées permettant à l'opérateur de voir s'il s'agit bien du livre qu'il a en main. De même, l'ordinateur émettra un signal sonore lorsqu'il inscrit un bien culturel dans une bibliothèque différente de celle de l'inscription précédente afin qu'on prenne soin de déposer le document dans la boîte.

En mode automatique, l'ordinateur choisit les bibliothèques par ordre de priorité. II vérifie s'il reste des biens à expédier à cette bibliothèque, si le pourcentage de nouveautés et la période de carence sont fixés, si le bien est dans la collection locale de la bibliothèque, s'il a fait l'objet d'une demande spéciale et s'il fait partie d'un document. Une fois en plusieurs volumes,jcet algorythme complété, il inscrit le document dans la bibliothèque et l'indique à l'écran.

La désinscription (provenance) se fait dans la même opération que l'inscription. Pour confirmer la destination, l'opérateur procède à la lecture du code à barres d'un autre document.

En tout temps, le préposé peut connaître, grâce à une clef de fonction appropriée, l'état de la rotation des collections d'une bibliothèque tant au plan des réceptions qu'à celui des expéditions.

\section{Confirmation du retour et de l'expédition}

Une BCP peut assurer un roulement des collections déposées de 0 à $100 \%$. Une BCP ayant 140000 articles culturels en dépôt rapportera à la centrale 112000 biens culturels et en expédiera autant avec un roulement de $80 \%$. II y a donc un contrôle nécessaire des échanges afin de s'assurer des quantités reçues et expédiées. Le système produit donc, par bibliothèque affiliée, un premier rapport sur les biens expédiés sous forme de liste d'expédition.

La liste d'expédition sert également de liste de nouveautés pour la bibliothèque affiliée. Cette liste assurera également la mise à jour temporaire du catalogue imprimé qui lui parviendra dix à 15 jours après la désinscription des volumes retournés par la bibliothèque affiliée. 


\section{Gestion des demandes spéciales}

Les demandes spéciales sont des requêtes de volumes faites par les bibliothèques affiliées auprès de la centrale. Elles permettent aux populations locales un accès à l'ensemble de la collection de la BCP. Le système des rotations permet de bloquer tout prêt d'un titre qui est en demande spéciale. Si le document arrive par un échange régulier le système indiquera à l'opérateur qu'il ne peut inscrire le document, car une bibliothèque l'a demandé.

On acheminera le document à la bibliothèque affiliée par le sous-module de confirmation d'envoi des demandes spéciales. Le système mettra à jour automatiquement l'inventaire de la bibliothèque affiliée. Lors du prochain échange, le système verra à rappeler les documents excédentaires d'une ou plusieurs catégories à la suite des demandes spéciales. De même, il rétablira l'équilibre de la bibliothèque prêteuse. La tenue à jour de l'inventaire est donc permanente.

Les BCP comptent à court terme ajouter des fonctions à ce sous-menu du module rotation. Elles voudraient que le système équilibre automatiquement les demandes faites et reçues d'une bibliothèque affiliée, qu'il choisisse la bibliothèque destinataire, qu'il fasse les rappels et compile desjstatistiques.

\section{Sous-produits du module de rotation}

Évidemment, si les bibliothèques centrales de prêt ont investi tant d'argent et d'efforts dans l'amélioration du processus de rotation ce n'est pas seulement en raison des gains de productivité au niveau des opérations d'inscription et de désinscription des biens culturels. Certes, un employé inscrit, et ce d'une façon plus efficace, quatre fois plus de documents à I'heure que dans le système manuel avec une marge d'erreur virtuellement inexistante. Mais il y a plus que ces gains réels.

\section{a) Les catalogues auteurs et titres des collections déposées}

Un des objectifs de l'informatisation était l'implantation des catalogues et des outils bibliographiques dans les bibliothèques affiliées et l'amélioration du soutien aux comités. Cet objectif a été réalisé à $100 \%$ à la $\mathrm{BCP}$ de la Montérégie. La totalité de ces 65 bibliothèques affiliées ont reçu au premier trimestre 1988 leurs catalogues auteurs et titres sous forme imprimée.

Nous avons retenu comme technique de production l'impression au laser. À partir d'un fichier ordinolingue produit par notre système, une compagnie spécialisée dans l'impression au laser, imprime et relie les catalogues de chaque bibliothèque. L'impression coûte environ $.007 \$$ par notice, ce qui représente pour une BCP, comme celle de la Montérégie, $5000 \$$ par an plus $3000 \$$ de traitement informatique.

La distribution des catalogues est gratuite et fait partie du service de base. En utilisant cette méthode plutôt que celle des fiches traditionnelles, c'est quelque 7000 heures de classement que nous évitons aux bénévoles pour la seule BCP de la Montérégie. Reporté sur l'ensemble des $B C P, c^{\prime}$ est 50000 heures de classement au niveau des bibliothèques affiliées et quelque 25000 heures au niveau des centrales.

La BCP de I'Abitibi-Témiscamingue offre, pour sa part, un catalogue imprimé A-T-Sujets suivant chaque rotation; la mention de la date de dépôt permet aux bénévoles de connaître l'ancienneté de leurs collections déposées et d'en tenir compte lors des rotations.

\section{b) Les catalogues sur microfiches}

La BCP de la Montérégie a commencé en avril 1988 la distribution des catalogues sur microfiches. Une adaptation du logiciel de production des microfiches de multiLIS fut nécessaire afin de rendre les microfiches plus faciles à consulter pour le grand public ; le module avait été développé à l'origine pour le réseau des collèges du Québec.

Le catalogue sur microfiches de la BCP Montérégie donne accès aux quelque 60000 titres qu'elle a en dépôt ou en magasin. Grâce à ce catalogue complet, les usagers sont en mesure de savoir rapidement si le réseau de la $B C P$ possède le titre désiré ; ce catalogue permet d'accélérer la livraison des demandes spéciales.

La parution des catalogues sur microfiches se fait trois fois par année. Un catalogue des vedettesmatière sur microfiches sera disponible au printemps 1989 . Ce type de catalogue sera grandement apprécié par les bibliothèques affiliées, puisqu'il permettra en outre l'accès à une documentation étoffée sur une grande variété de sujets.

\section{c) Traitement des collections locales}

L'ampleur qu'ont voulu donner la BCP Montérégie et celle de l'Abitibi-Témiscamingue aux collections locales depuis quelques années a porté fruit. Les bibliothèques affiliées à ces deux $B C P$ possèdent 80000 biens culturels dans leurs collections locales. Tout en y prenant goût, les 
bibliothèques se rendent rapidement compte qu'il faut les organiser si on veut bien les utiliser.

Au cours des été 1987 et 1988, la BCP Montérégie a tenté quatre expériences pilotes de traitement des collections locales sur son ordinateur central. On a fait modifier le programme de rotation pour tenir compte des collections locales lors de l'inscription. Pour sa part, la BCP de l'AbitibiTémiscamingue offre depuis le printemps 1987 le traitement informatique des collections locales et des collections pour le service au public. Les bibliothèques qui commencent à développer des collections locales importantes devront en envisager sérieusement le traitement sur l'ordinateur de la centrale. D'ailleurs, le développement de collections-réseau sera l'une des prochaines préoccupations des BCP.

\section{d) Interrogation en direct}

La BCP Montérégie a amorcé, en septembre 1987. une expérience conjointe d'interrogation en direct de sa banque de données bibliographiques. La bibliothèque municipale de Richelieu avait accepté avec empressement de mener à bien cette expérience pilote.

Au cours des mois d'octobre, novembre et décembre, cette bibliothèque municipale a fait 61 demandes spéciales de documents à nos services. L'an dernier, cette bibliothèque avait fait 59 demandes sur une période de 12 mois. On peut donc conclure que le nombre de demandes spéciales a augmenté substantiellement grâce à l'accès par terminal à la banque de données de la centrale. En fait, si nous extrapolons, la bibliothèque de Richelieu ferait entre 150 à 200 demandes en douze mois, soit une augmentation de $400 \%$.

Les bénévoles ont, de plus, complété quelque 23 heures d'interrogation. Ils ont effectué au cours de ctte période 212 recherches. Celles-ci ont permis de répondre immédiatement à l'usager, de trouver de l'information qui autrement n'aurait pas été disponible et, enfin, de placer des demandes spéciales avec la quasi-certitude que la $B C P$ possède le document. Donc, une amélioration notable du service.

Quant aux coûts, outre les frais de service pour la ligne téléphonique, ils semblent réduits au minimum. D'ailleurs, si on place 100-150 demandes spéciales de plus par année à la BCP, ceci équivaut à une valeur économique de 2000 \$ à $3000 \$$ en biens culturels. II y a donc une grande rentabilité à utiliser l'accès en direct.

Grâce aux terminaux locaux, il sera possible éventuellement aux bibliothèques d'acheminer directement leurs demandes spéciales et de signaler leurs achats locaux. Nous éviterons ainsi de leur expédier des volumes déjà disponibles dans leurs collections locales. Nous pourrons également imprimer leurs catalogues en leur fournissant les données catalographiques nécessaires au traitement matériel de leurs documents.

\section{e) Échanges de biens culturels personnalisés}

Un des objectifs de l'informatisation de la BCP était également de permettre des échanges de documents mieux adaptés aux besoins des bibliothèques. Ainsi, il sera possible d'échanger une catégorie plus rapidement qu'une autre en y attribuant un taux de rotation plus élevé. À titre d'exemple, on pourra échanger $150 \%$ de la collection de romans en un an, tandis qu'on échangera seulement $20 \%$ de la collection en sciences sociales; ainsi chaque bibliothèque pourra gérer une collection adaptée au profil distinct de sa propre clientèle.

\section{Perspectives d'avenir}

Les BCP ont regroupé une bonne partie de leurs achats de biens culturels auprès de deux BCP soit I'Outaouais et la Mauricie. Celles-ci font office de centrale de choix, d'acquisitions, de traitement et de préparation matérielle auprès des BCP clientes. Le catalogage sur fiches $3 \times 5$ devient vite un handicap lorsqu'on veut procéder à l'échange d'information catalographique dans un système automatisé; la centrale expédie les données catalographiques sur fiches et la BCP cliente doit les intégrer à son ordinateur.

Les $B C P$ ont décidé de réduire cette contrainte en développant un module de transfert électronique des données qui procèdera au téléchargement des notices d'une centrale de service à ses bibliothèques clientes; tout en réduisant les délais, on éliminera également la multiplication des saisies de données par les BCP.

Ce module de téléchargement, intégré à multiLIS, permettra également à une BCP d'offrir aux bibliothèques autonomes de sa région qui utilisent multiLIS la possibilité de repiquer lesjdonnées catalographiques de la BCP. II sera désormais possible pour les BCP intéressées d'envisager la mise sur pied de centrales de service au niveau régional.

Une des exigences inhérentes au transfert électronique des données a été l'obligation pour les BCP de normaliser leurs principes et procédures de traitement. Les BCP se sont dotées de règles communes de saisie de données, de catalogage, de classement et d'analyse documentaire. Cette normalisation est essentielle au 
développement harmonieux d'une opérationréseau.

Certes les BCP, en offrant les catalogues imprimés, les catalogues sur microfiches, le traitement des collections locales et l'interrogation en direct, ont révolutionné les outils d'accès à l'information pour leurs bibliothèques affiliées. Toutefois, il reste encore plusieurs améliorations à apporter à la gestion locale de ces bibliothèques.

Qu'elle desserve 1000 ou 5000 habitants, une bibliothèque affiliée est aux prises avec les mêmes problèmes de fonctionnement qu'une bibliothèque autonome. Il faut que son personnel, rémunéré ou bénévole, assure l'information, le prêt, les réservations, les réclamations, les statistiques et le traitement des collections locales. Certaines BCP entrevoient pousser plus loin l'intégration technologique des bibliothèques affiliées. Elles entendent faire adapter en partie le logiciel multiLIS à la gestion des bibliothèques affiliées. Un «micromultiLIS » fonctionnant sur un micro-ordinateur assurerait les opérations de prêts, réservation, interrogation et traitement. La $\mathrm{BCP}$ expédierait aux bibliothèques une mise à jour sur disquette de la collection déposée plutôt que sur une copie papier.

Le $C D / R O M$ est une autre innovation technologique susceptible d'aider les BCP à mieux remplir leur mandat. On peut entrevoir que la BCP publiera son catalogue collectif sur disque au laser dès que cette technologie sera disponible à un coût abordable. Le CD/ROM viendrait remplacer, pour certaines bibliothèques dont l'importance le justifierait, le catalogue sur microfiches. L'accessibilité directe à cette technologie pour le public ouvre une exploitation insoupçonnée des ressources documentaires.

\section{Conclusion}

En 1979, la BCP de l'Abitibi-Témiscamingue entrevoyait l'utilisation de l'informatique pour rendre davantage accessible la lecture aux milieux à faible densité de population. II s'agissait dans un premier temps d'automatiser les centrales. La technologie a, depuis dix ans, évolué au point qu'elle est maintenant à la portée des bibliothèques affiliées.

Certes, il reste beaucoup à faire pour que les huit cents bibliothèques affiliées à l'une ou l'autre des onze BCP du Québec réalisent cet objectif. Les expériences pilotes des BCP de l'AbitibiTémiscamingue et de la Montérégie ont permis la réalisation d'un système commun à l'ensemble des $\mathrm{BCP}$, préalables à un véritable réseau national de gestion documentaire; de plus, I'application technologique aux bibliothèques affiliées, partageant un système commun, offre un potentiel virtuellement inexploré de systèmes documentaires aux milieux à faible densité démographique.

\section{VOTRE SERVICE \\ EXPRESS Datowewentrit EXPRES LEANADA

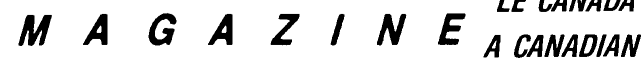 Une division de E.M. Press inc. SERVICE}

- Spécialiste des revues françaises au Canada depuis 7 ans.

- Plus de 1000 titres de périodiques français, italiens, espagnols.

- Service personnalisé aux bibliotheques (information des éditeurs, réserve de numéros).

- Gestion informatisée des titres.

- Expédition des revues de Montréal

Une division de E.M. Press 4001 A, boulevard Robert. Montréal, Québec H1Z 4 H6 Canada Tél. . (514) 374.9811 1-800-363-7141
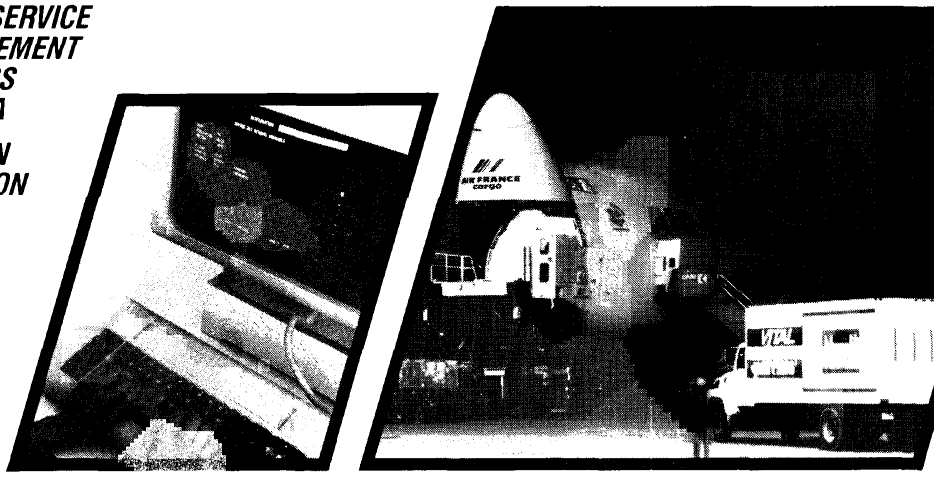

1.800 .363 .7141


Figure 1

Menu principal du système multiL/S

\section{«Système multiLIS menu principal}

Catalogage

Prêt

Acquisitions

Rotation

Rapports

Contrôle des imprimantes

Départ des centraux

Arrêt des centraux

Bureautique
Figure 2

Menu principal du système de rotation

\section{Système de rotation des collections}

1 - Gestion des bibliothèques affiliées

2 - Gestion des quantités à ajouter

3 Gestion des catégories par type de biens culturels

4 - Initialisation des rotations

5 - Rotation des collections

6 - Confirmation de retour d'une bibliothèque affiliée

7 - Confirmation d'expédition d'une bibliothèque affiliée

8 - Confirmation d'envoi de demandes spéciales

9 - Statut des bibliothèques affiliées

10 - Statut de la gestion des bibliothèques affiliées

11 Lecu fichier des notices

Figure 3

\section{Gestion des bibliothèques affiliées}

\section{Gestion des bibliothèques affiliées}

Numéro de la bibliothèque affiliée :

Adresse d'expédition

1. Nom:

2. Adresse 1 :

3. Adresse 2:

4. Code postal:

5. Téléphone:

Informations complémentaires:

11. Responsable:

12. Répondant:

15. Types de bien

16. Population

17. Collection locale

20. Remarques:

Statut de rotation: Numéro de rotation en cours :

21. Nombre de rotation / année:

22. Priorité de rotation:

Numéro à modifier :
Adresse contact (Référence)

6. Nom:

7. Adresse 1:

8. Adresse 2:

9. Code postal:

10. Téléphone:

13. Responsable DS :

14. Téléphone D.S. : 999-9999

18. Année:

19. Cycle: 
Figure 4

Gestion des quantités par type de biens culturels

Gestion des types de biens culturels

Numéro de la bibliothèque : 20 Saint-Rémi Type de biens culturels: VOL Volumes

Équilibre constaté : 100

Quantité globale : $\mathbf{5 0 0 0}$

1 - Quantité à ajouter: 200 Sur quelle catégorie: Romans

2 - Taux de rotation annuel: $90 \%$

Numéro à modifier
Figure 5

\section{Gestion des catégories par type de biens culturels}

\section{Gestion des catégories par type de biens culturels}

Numéro de la bibliothèque affiliée : 20 Saint-Rémi

Type de biens: VOL Volumes français

Catégorie : 100 Adultes documentaires

Quantité globale: 250

1. Période de validité des nouveautés : $\mathbf{2 4}$ mois

2. Période de validité du dépôt : $\mathbf{3 6}$ mois

3. Équilibre constaté: $\mathbf{5 . 3 5}$ 4.Equilibre visé : 5.00

5. Taux de rotation : $\mathbf{4 8} \%$

6. \% de nouveautés: $20 \% \quad 7$. Obligatoire $(O / N)$ : Oui

8. Collection locale $(\mathrm{O} / \mathrm{N})$ : Oui

Numéro à modifier : $X$
Figure 6

Initialisation de la rotation

Initialisation de la rotation

Numéro de la succursale : 20

Numéro de la rotation : 4

Statut actuel : INACTIF

4 Nouveau Statut:

5 Date de l'échange : 12-08-88

6 - Heure de l'échange : 10:00
Figure 7

Inscription des biens culture/s

Inscription des biens culturels

Succursales en rotation : $2,4,8,56,34,15,26$, $54,61,39,16.1$

Mode d'inscription : automatique

Numéro de document : 30001001640665

Titre: Arthur et Jérémie Type de bien: VOL

Auteur: Smith, Doris Catégorie: B.D.

Cote:

Nouveauté : OUI

Provenance: 9 Destination : * 
Figure 8

État d'avancement de l'expédition

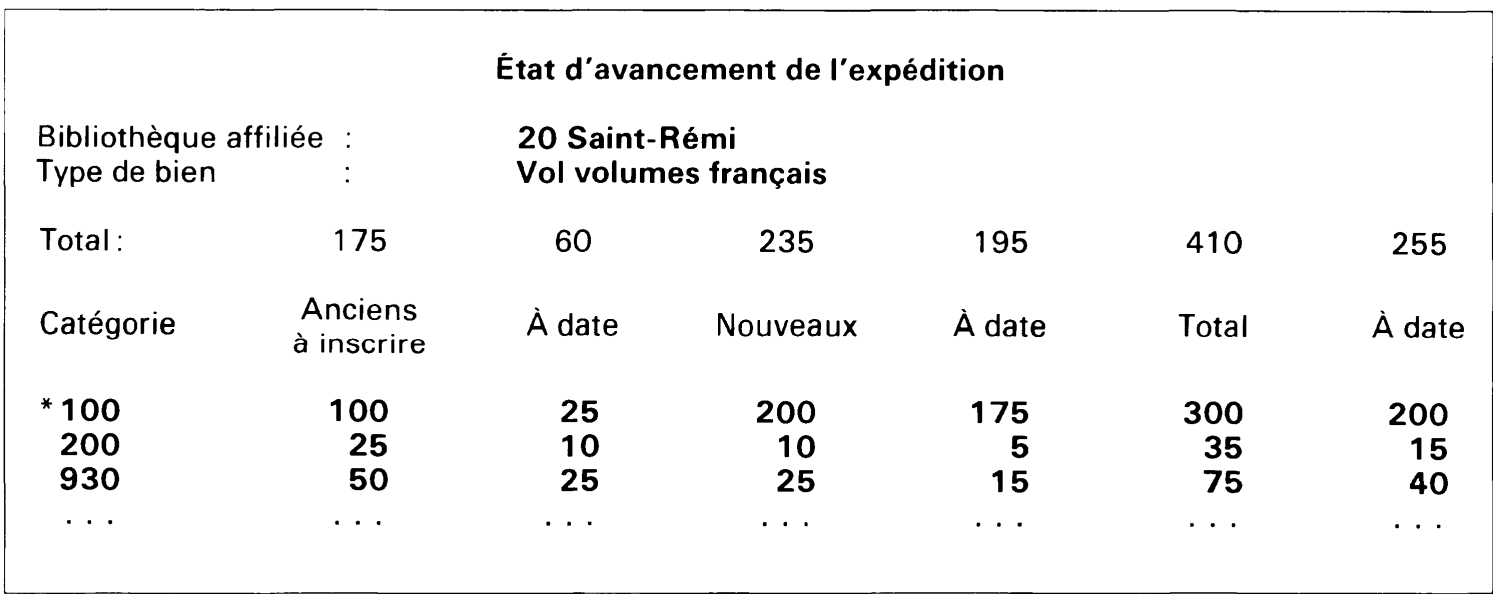

Figure 9

État d'avancement de la réception

\begin{tabular}{|c|c|c|c|}
\hline \multicolumn{4}{|c|}{ État d'avancement de la réception } \\
\hline $\begin{array}{l}\text { Bibliothèque affiliée } \\
\text { Type de bien }\end{array}$ & $\begin{array}{l}20 \text { Saint-A } \\
\text { VOL Volun }\end{array}$ & & \\
\hline Total : & 500 & 400 & 100 \\
\hline Catégorie & À recevoir & Reçus & Écart \\
\hline $\begin{array}{r}* 100 \\
200 \\
300 \\
\text { B.D. }\end{array}$ & $\begin{array}{r}100 \\
50 \\
200 \\
150\end{array}$ & $\begin{array}{r}75 \\
10 \\
200 \\
100\end{array}$ & $\begin{array}{r}25 \\
40 \\
0 \\
50\end{array}$ \\
\hline$\cdots \cdots$ & $\cdots$ & $\ldots \ldots$ & $\cdots \cdots$ \\
\hline
\end{tabular}

Figure 10

Confirmation d'envoi d'une demande spéciale

Confirmation d'envoi d'une demande spéciale

Titre:

Auteur:

Cote :

Numéro de bibliothèque :
Réponds gentiment mon petit Janikovsky, Eva

ALB

9 Saint-Marc-sur-Richelieu 


\section{AVIS DE ROTATION}

Numéro et nom de bibliothèque: St-Jean-Baptiste $N^{\circ} 42$

Rotation numéro: 11

Date de la livraison: $\quad$ Mercredi, 16 novembre 1988

Heure de la livraison: 11 hres 30

Coordonnatrice (eur) : $\quad$ Madame Louise Desjardins

Adresse: $\quad 4610$, rang ds Soixante

St-Jean-Baptiste, Québec

JOL 2BO

Type de document : Volume (VOL)

$\begin{array}{lc}\text { Catégorie } & \text { Nombre à retourner } \\ 100 & 26 \\ 13 \text { (Romans adultes) } & 170 \\ 200 & 6 \\ 300 & 25 \\ 400 & 0 \\ 500 & 0 \\ 600 & 38 \\ 700 & 29 \\ 800 & 19 \\ 900 & 12 \\ 920 & 26 \\ 930-999 & 20 \\ 99 \text { (000-099) } & 1 \\ \text { BD } & 77 \\ \text { JA } & 89 \\ \text { JD } & 57 \\ \text { JR } & 46\end{array}$

Total : 641

Type de document: DISQUE (DIS)

Catégorie

C

D

E

$\mathrm{PI}$

PV
Nombre à retourner

8
3
1
2
17

Total : 31 